\title{
De los outsiders de Norbert Elias y de otros extraños en el campo de la sociología de las migraciones
}

\author{
Rosalina Alcalde Campos \\ Departament de Sociologia. Universitat Autònoma de Barcelona \\ Rosalina.Alcalde@uab.cat
}

\section{Resumen}

En el año 1965, Norbert Elias y John L. Scotson publicaron The established and the outsiders: A Sociological Enquiry into Community Problems. En esta obra, los autores tratan una problemática sociológica ampliamente estudiada desde el campo de la sociología de las migraciones: cómo se construye socialmente la figura del forastero o del extraño. Con todo, no son muy abundantes las referencias a esta obra en la producción científica española relativa al estudio de los procesos de construcción de alteridades en sociedades receptoras de inmigración como la nuestra. Aunque, de igual modo, resulta significativo que tampoco Elias y Scotson hagan referencia a estudios clásicos sobre esta materia. Por todo ello, en este artículo, se tratará de relacionar la investigación de Elias con la de autores antecesores en este tipo de trabajos, para, finalmente, destacar qué nos aporta este libro para el estudio de los asentamientos de población inmigrada y sobre las relaciones entre e intra distintos grupos sociales.

Palabras clave: extranjero; inmigrante; alteridad; inmigración; estigma.

\section{Abstract: On Norbert Elias' outsiders and other strangers in the sociology of migration}

In 1965, Norbert Elias and John L. Scotson published The established and the Outsiders: A Sociological Enquiry Into Community Problems. In this work, the authors discuss a sociological problem widely studied from the sociology of migration: how the figure of the foreigner or stranger is constructed socially. However, references to this book in Spanish scientific production about the study of the processes of construction of otherness in immigration receiving societies like ours are not very abundant. Yet it is significant that Elias and Scotson also do not make references to classic studies on this subject. Therefore, this article will attempt to link the research of Elias whit the work of his predecessors within this field of study. Finally it tries to highlight what the outcome of this book is for the study of the settlement of immigrant population and the relationships between and within social groups.

Key words: foreign; immigrant; otherness; immigration; stigma. 


\section{Sumario}

\begin{tabular}{|c|c|}
\hline 1. Introducción & 4. La estigmatización del outsider: \\
\hline $\begin{array}{l}\text { 2. Las relaciones entre outsiders y } \\
\text { established en la pequeña comunidad de }\end{array}$ & \\
\hline $\begin{array}{l}\text { tablished en la pequeña comunidad de } \\
\text { Wiston Parva }\end{array}$ & \multirow{2}{*}{$\begin{array}{l}\text { Conclusiones. Sugerencias para } \\
\text { la investigación en el campo } \\
\text { de la sociología de las migraciones } \\
\text { desde la lectura de Elías }\end{array}$} \\
\hline \multirow{2}{*}{$\begin{array}{l}\text { 3. Los otros outsiders en el campo de la } \\
\text { sociología de las migraciones: el extranjero, } \\
\text { el hombre marginal y el forastero }\end{array}$} & \\
\hline & Bibliografía \\
\hline
\end{tabular}

\section{Introducción}

Una obra que ha sido titulada The established and de outsiders (1994) (primera edición: 1965) evoca, de forma casi instantánea en una investigadora de la sociología de las migraciones, la cuestión de la construcción social de la figura del extraño o extranjero. Y sugiere su lectura desde una mirada particular, por más que Elias nos remita en su estudio a la descripción de cómo se configuran las diferencias entre establecidos y forasteros, entre vecinos y vecinas de una misma nacionalidad en una pequeña localidad: Winston Parva.

Esta obra de Elias y Scotson aborda una cuestión que ya viene siendo tratada por las ciencias sociales desde el siglo XIX, a saber, cómo se establecen las relaciones sociales entre determinados grupos en base a la estigmatización.

\section{Las relaciones entre outsiders y established en la pequeña comunidad de Winston Parva}

El estudio de Elias y Scotson se sitúa en una pequeña comunidad suburbana donde se diferencian dos comunidades. Una, las más antigua en su asentamiento y que reside en el centro de la localidad, otra, de más reciente llegada, instalada en los alrededores. El interés por el estudio de esta comunidad vino dado en un inicio por el hecho de que uno de los vecindarios presentaba un elevado grado de delincuencia y bajo estrato social, por lo que los autores se propusieron explicar el grado diferencial de delincuencia entre los suburbios de esta localidad y su correlación con las características de los barrios y las relaciones entre sus vecinos. Pero lo que encontraron los investigadores fue un hecho que arrojó luz sobre un problema que, a menudo, se encuentra a una escala mucho mayor que la de este lugar. Tras tres años de investigación, se constató que las diferencias entre el nivel de delincuencia de los dos barrios más grandes prácticamente eran inexistentes. Pero, a pesar de ello, entre los habitantes del barrio en que vivía la comunidad que llevaba más tiempo asentada en Winston Parva, persistía el estigma acerca de sus vecinos.

Por dichos motivos, finalmente, los autores reorientaron su objeto de estudio y se centraron en explicar cómo, en esta pequeña comunidad, los miembros que llevaban más tiempo establecidos trataban a los recién llegados de tal forma que lograban estigmatizarlos como grupo con menos virtudes humanas, en cambio, el grupo de los established se atribuía unas virtudes humanas 
superiores. Lo que consigue explicar el hecho de que, en esta comunidad, un grupo llegara a distinguirse como establecido en contraposición al de los forasteros eran los recursos de poder de que disponía cada uno de ellos. A mayor desigualdad de poder, menos realismo y más distorsión se daba sobre la percepción de los establecidos, si bien el elemento más novedoso de este análisis es el reconocimiento de que el poder no venía dado, en este caso, por los recursos materiales, de poder o clase social, nacionalidad o pertenencia racial o étnica, sino que se fundamentaba en el orden de llegada, es decir, en la antigüedad en el establecimiento. Luego el poder del grupo venía dado por el grado de cohesión de éste. Es así como, en el caso de la comunidad de Winston Parva, la circunstancia que justificaba la atribución de la exterioridad (los outsiders) era el tiempo de llegada a la comunidad. Los «establecidos», el grupo con poder, cerraban filas entre sus miembros y estigmatizaban a los «forasteros» como carentes de virtud humana suprema y, por tanto, del carisma distintivo que el grupo dominante se atribuía a sí mismo. Los autores explican cómo los miembros del grupo dominante mantenían la convicción de ser no sólo los más poderosos, sino también mejores seres humanos que los miembros del otro grupo. Para imponer esta creencia sobre su propia superioridad, trataban a las personas recién llegadas como desarraigadas, como forasteras.

Los recién llegados, además de ser tratados como forasteros y desarraigados, eran, también, excluidos de todo contacto social no ocupacional con los miembros del otro grupo y, al poco de instalarse, parecían aceptar con una resignación desconcertante su pertenencia a un colectivo de menor virtud y respetabilidad. El tabú que rodeaba a este tipo de contactos se preservaba mediante mecanismos de control social, tales como el cotilleo elogioso acerca de los que respetaban el tabú y la amenaza de cotilleo condenatorio dirigido contra supuestos transgresores.

En esta comunidad, la superioridad de poder del grupo establecido desde antaño descansaba en el alto grado de cohesión de las familias, que se conocían entre sí desde hacía dos o tres generaciones, en contraste con los recién llegados, que eran extraños no sólo en relación con los viejos residentes, sino también entre sí. El mayor potencial de cohesión, junto con los medios de control social, hacía posible que los viejos residentes perpetuaran un acceso desigual a los puestos dirigentes de las organizaciones locales (ayuntamiento, iglesia, club), excluyendo de ellos a los vecinos del otro barrio, grupo mucho menos cohesionado. La exclusión y la estigmatización de los forasteros por parte de los establecidos lograban preservar la identidad del grupo, afirmaban su superioridad y mantenían a los demás en un lugar periférico.

En este sentido, Elias y Scotson adoptan un enfoque figuracional para comprender y explicar las condiciones en que un grupo está en disposición de difamar a otro grupo a través de las sociodinámicas de la estigmatización. La aportación de esta investigación radica en elevar la perspectiva del prejuicio individual a la del nivel del grupo (estigmatización grupal). El eje de esta figuración es el desequilibrio del poder y las tensiones inherentes a él. Al mismo tiempo, otra aportación relevante ha sido la de insistir en que, si bien el estigma puede estar 
configurado por elementos culturales, raciales o étnicos, su base tiene origen en las relaciones de poder y en los procesos de exclusión o desigualdad social.

Así, el estudio realizado en Winston Parva describe de qué manera se produce la estigmatización de los recién llegados, lo que nos evoca paralelismos acerca de cómo las sociedades receptoras de inmigración consolidan el imaginario sobre la figura del inmigrante.

\section{Los otros outsiders en el campo de la sociología de las migraciones: el extranjero, el hombre marginal y el forastero}

El tema abordado en la investigación de Elias y Scotson es relevante, y la literatura sociológica y geógrafa identifica numerosas tipologías de «extraños», muchas veces superpuestas, que incluyen también a los marginados, ya sean individuos o grupos, a las subculturas urbanas y a las minorías étnicas, si bien una parte importante de esta producción literaria, en especial desde la sociología urbana, se ha dedicado a explorar las relaciones entre las sociedades de acogida y las personas recién llegadas. Por lo general, estas últimas son inmigrantes (Alexander, 2003).

Quizás una de las cosas más sorprendentes a la hora de indagar en esta obra de Norbert Elias (Breslau, Alemania (actualmente Wroclaw, Polonia), 1897 Amsterdam, 1990) ${ }^{1}$ y John L. Scotson, y sobre todo al hacerlo con la expectativa de relacionarlo con los estudios sobre alteridad e inmigración, ha sido comprobar las escasas referencias a este autor precisamente en este tipo de trabajos, al menos a lo que en el caso español se refiere. Pero, de igual forma, la omisión por parte de Elias y Scotson de obras anteriores a su investigación y que abordaron de forma pionera el análisis de la figura del marginado, el inmigrante o el extranjero como figuras de alteridad es, así mismo, paradójico, principalmente si consideramos que algunos de estos autores no sólo fueron predecesores y/o coetáneos de Elias, sino, al igual que él, también europeos, algunos de ellos con interesantes paralelismos con la propia historia de vida del autor de The stablished and the outsiders. Al igual que Elias, la condición de judíos, como es el caso de Simmel, y de judíos exiliados, como fue el caso de Schützs, explica en gran parte el interés de estos científicos sociales por el análisis de los procesos de estigmatización y de alteridad entorno a las figuras del extranjero, del extraño o del outsider. De hecho, pueden intuirse en esta obra las propias vivencias de Elias en relación con la marginación que el pueblo judío ha vivido en ámbitos como la universidad, la diplomacia y la cultura (Béjar, 1994).

Cuarenta años antes de la publicación de la obra a la que aquí nos referimos, Georg Simmel (Berlín, 1858-Berlín, 1928) publicó, en 1927 y de forma pionera, su brillante ensayo titulado Digresiones sobre el extranjero. Predecesor y profesor de R.E. Park, tomó la ciudad, la urbe, como el espacio en el que analizar las relaciones humanas, distinguiendo éste de localidades pequeñas en

1. Web Norbert Elias Foundation: http://www.norberteliasfoundation.nl/ 
que las relaciones son más comunales. Simmel reconoce en la metrópoli tanto un elemento liberador para los individuos, como la cuna de producción de nuevos modos básicos de interacción social, lo que conduce a la libertad del individuo. La ciudad determina la intelectualización y la superficialidad de los contactos urbanos; una forma funcional de interacción que Simmel defendió siempre como estrategia utilizada por los urbanitas para enfrentarse a la realidad fragmentaria, fugaz y racionalizada de las grandes ciudades (De la Peña, 2003). Es desde este marco geográfico de análisis donde los sociólogos de la Escuela de Chicago van a insertar sus estudios sobre las interacciones entre inmigrantes y autóctonos o establecidos y que representa una divergencia clara con el estudio de Elias, que vuelve a situar el análisis en la comunidad.

Años más tarde, Robert Ezra Park (Pennsylvania, 1864-Tennessee, 1944) desarrollaría ampliamente, desde el Departamento de Sociología de la Escuela de Chicago, trabajos orientados a la cuestión del inmigrante en los Estados Unidos. Destaca, en este sentido, su obra Human Migrations and the Marginal Man (1928). Park hereda su concepción de las relaciones étnicas que marcan las interacciones entre inmigrantes y nativos del planteamiento de Williams Isaac Thomas y Florian Znaniecki esbozado en su obra The polish peasant in Europe and America (1918), si bien, como señala Martínez (2000), sus interpretaciones están fuertemente influenciadas por William James, Josiash Royce, John Dewey y, especialmente, por su maestro, Georg Simmel.

Para Park —y en sintonía con Simmel—, la ciudad logra configurar múltiples grupos y hace posible que los individuos puedan moverse entre estos y rehuir así las constricciones del grupo primario, los vínculos locales. Esto otorga al ambiente urbano una diversidad social y cultural, una libertad que estimula y educa a los ciudadanos en nuevas referencias de comportamiento posible, lo cual facilita la aparición del hombre marginal (Martínez, 2000).

En las ciudades el viejo clan y los grupos de parentesco se han disuelto y han sido reemplazados por una organización social basada en intereses racionales y en preferencias temperamentales. De un modo más concreto, la gran división del trabajo que rige en las ciudades permite y más o menos obliga al hombre individual a concentrar sus energías y su talento en la tarea específica que mejor desempeña, y en este sentido él y sus compañeros se emancipan del control de la naturaleza y de las circunstancias que tan rigurosamente dominan al hombre primitivo. (Park, 1928)

Todo lo contrario a lo que ocurre en Winston Parva, donde las personas marginadas no deciden serlo, sino que esa condición les es impuesta por el grupo de los established, donde su posición marginal no es un caso de libertad, sino una condena estigmatizadora. La figura del outsider de Elias está sujeta a una comunidad que la configura y la construye como tal. No es un ser errante y movible como el hobo o el hombre marginal de Park. Los recién llegados a Wiston Parva llegan para instalarse allí, quieren ser sedentarios, si bien el estigma los convierte en perpetuos forasteros, casi como si fueran extranjeros en su tierra. 
Si la emigración — dice Simmel—, considerada como no vinculación a un punto dado en el espacio, constituye el concepto opuesto a la sedentariedad en un punto cualquiera, entonces, con toda seguridad, la forma sociológica del extranjero representa la unión de ambas determinaciones.

El extranjero permanece pero no llega a asentarse, no es sino un emigrante en potencia. Esto significa que no se encuentra ligado a los otros por normas y convenciones locales.

Es un hombre libre, en lo práctico y en lo teórico. Considera su relación con los otros con menos prejuicios; los somete, pues, a un patrón más general y objetivo, y las tradiciones, las costumbres o los afectos no limitan su acción. (Park, 1914)

$\mathrm{Al}$ igual que este extranjero de Park, los outsiders de Elias, al no formar parte de un grupo, disfrutan de alguna manera de ese grado de libertad, pero, a cambio, se ven relegados a una posición social periférica dentro de la sociedad de Wiston Parva. Ahora bien, los privilegiados, los established pagan un precio alto por su condición, a saber, la de verse sujetos a las normas que marca el grupo.

Con todo, la ciudad de Park y Simmel no consigue invisibilizar de igual modo a todos sus extraños. La invisibilidad del hobo o del hombre marginal que señala Park no se da entre aquellos inmigrantes o extraños que sustentan rasgos fenotípicos que los identifican.

[...] el japonés porta en sus rasgos un sello racial distintivo; lleva, por así decirlo, un uniforme racial que lo clasifica. Él no puede llegar a ser un simple individuo, imperceptible entre la masa cosmopolita, como ocurre por ejemplo con el irlandés, y en menor medida, con otros grupos raciales inmigrantes. El japonés, como el negro, está condenado a permanecer entre nosotros como una abstracción, como un símbolo - y no sólo como símbolo de su propia raza sino de todo Oriente y de esa tan imprecisa e indefinida amenaza a la que algunas veces nos referimos como «el peligro amarillo». (Park, 1914)

En este sentido, un hecho destacable de las dinámicas de estigmatización descritas por Elias y Scotson es, precisamente, la inexistencia de elementos visibles que permitan identificar y diferenciar a un grupo de otro en esta pequeña comunidad. Aparentemente, nada les diferencia: misma clase social, rasgos fenotípicos iguales, misma nacionalidad e idioma... Y, por tanto, las señales del estigma son mucho más sutiles.

Otros sociólogo pionero en este tipo de estudios fue Alfred Schütz (Viena, 1899-Nueva York, 1959), también de origen judío, con su publicación, en 1944, de su artículo "El forastero». Esta obra, que inquiere en la misma línea que la de Simmel, considera que la condición de átopos del inmigrante como persona libre representa una amenaza para la cohesión social del grupo, lo que hace considerar al extranjero como un enemigo de la comunidad. Schütz sostiene que el pensar habitual puede ser mantenido mientras confirmen su vali- 
dez ciertos supuestos básicos. Si uno sólo de estos supuestos deja de confirmarse, se produce un derribo brusco del sistema de significatividad, con lo cual se revela el hecho de que la pauta general y su aplicabilidad se limita a una situación histórica específica. El forastero de Alfred Schütz — como el extranjero de Simmel, el hombre marginal de Park y el outsider de Elias - no comparte los supuestos básicos mencionados, cuestionándolo todo y haciendo vulnerable la cohesión del grupo. Desde el punto de vista del grupo al que se incorpora, él es un hombre sin historia. En definitiva, es peligroso para la integridad del grupo y también para su seguridad (Santamaría, 1994).

El paralelismo entre cómo es percibido y construido el imaginario del extranjero y cómo y por qué motivo se definen los outsiders de Winston Parva es notable, ya que tanto Simmel como Schütz revelaban ya entonces que la cohesión del grupo de los establecidos, fundada en el hecho de compartir una serie de valores y normas, provocaba un cierre de filas de estos para preservar su identidad y su cohesión. El instrumento que hace esto posible es la estigmatización del otro.

\section{La estigmatización del outsider: paralelismos con el extranjero}

Parece constatable que, entre las décadas de 1930 y 1960, diferentes investigadores del campo de las ciencias sociales y de la sociología a uno y otro lado del Atlántico se plantearon una perspectiva de estudio más cercana a la fenomenología, el interaccionismo simbólico y el socioconstructivismo, para dar respuesta a cuestiones que tienen que ver en cómo se dan determinadas lógicas de discriminación en los procesos de asentamiento de la población extranjera en las sociedades receptoras, considerando a su vez la configuración de las identidades grupales. Tal y como se ha visto, un elemento clave en la obra de Elias y Scotson es el análisis del estigma como instrumento que permite configurar y definir las características del grupo de los outsiders. Casi al mismo tiempo en que Elias y Scotson realizaban su investigación, Erving Goffman (Manville, Alberta, 1922-Filadelfia, Pennsilvania, 1982) publicaba, en 1963, Stigma: Notes on the Management of Spoiled Identity, después de haber publicado ya, en 1950, The Presentation of Self in Everyday Life, obra de referencia del interaccionismo simbólico. Gran parte del análisis de Elias y Scotson es igualmente llevado a cabo por Goffman. Dentro de los tres tipos de estigma señalados por este sociólogo (abominaciones físicas, defectos del carácter del individuo y tribales (raza, nación y religión)), sería el defecto del carácter el tipo de estigma utilizado por el grupo de los established para definir al de los outsiders. Goffman y Elias coinciden en que la aplicación del estigma deshumaniza a aquel a quien se le impone, lo que opera de igual forma en las categorías del outsider, del extraño, del forastero y del inmigrante.

Creemos por definición, desde luego, que la persona que tiene un estigma no es totalmente humana. Construimos una ideología del estigma, una ideología para explicar su inferioridad y dar cuenta del peligro que representa esa 
persona, racionalizando a veces una inoxidad que se basa en otras diferencias, como, por ejemplo, la clase social. En nuestro discurso cotidiano utilizamos como fuente de metáforas e imágenes términos específicamente referidos al estigma, tales como inválido, bastardo y tarado, sin acordarnos, por lo general, de su significado real. (Goffman, 2003: 15)

Refiriéndose a la persona estigmatizada, Goffman señala, al igual que lo hacen Elias y Scotson, cómo el sujeto al que se le aplica el estigma lo interioriza como elemento que configura su propia identidad.

El individuo estigmatizado tiende a sostener las mismas creencias sobre la identidad que nosotros; este es un hecho fundamental. (Goffman 2003:18)

En este sentido, el sujeto estigmatizado puede responder a esta situación de diferentes modos: corrigiendo su condición o, en el caso de los jóvenes outsiders de Winston Parva, cumpliendo las expectativas sociales que los demás tienen respecto a ellos: el fracaso escolar y el gamberrismo. En el caso de Winston Parva, los forasteros acababan por verse menos capacitados respecto a las normas de sus opresores y, por tanto, terminaban por experimentarse como individuos de valor inferior. Esta situación se traducía en apatía paralizante o en norma agresiva y en desorden.

En este punto, el análisis de Elias es relevante a la hora de analizar los procesos de asentamiento de la población inmigrada y las relaciones de convivencia entre autóctonos y extranjeros, ya que sostiene que las tensiones y los conflictos entre los dos grupos pueden permanecer en estado latente - cuando el diferencial de poder es enorme- o pueden manifestarse en forma de conflictos constantes, lo que sucede cuando el equilibrio de poder se traslada a favor de los extranjeros. Con relación a cómo los estigmatizados reaccionan frente al estigma, y aplicando este análisis a la inmigración extranjera en España, podríamos establecer paralelismos con las estrategias identitarias desarrolladas por los grupos de extranjeros establecidos y recién llegados (de borrado, de anclaje, mixtas, de transición (Colectivo Ioé, 1995, 1998), así como la cuestión de que la igualdad formal de derechos entre españoles y extranjeros no tiene porqué traducirse en ausencia de conflicto social.

En definitiva, como afirma Bauman (1995), todas las sociedades producen su propia clase de "extraños», y lo hacen de una forma inimitable. En el caso español, como en el europeo, la categoría de "extraño», del otro, se aplica generalmente a la de inmigrante y extranjero. Es por ello que la investigación de Elias y Scotson resulta de gran interés para plantear cómo alguien llega a ser definido, pero también a autodefinirse, como un forastero, un extranjero, un inmigrante... Un outsider.

En el caso de la figura del extranjero, esta distinción viene marcada por la configuración hegemónica de las sociedades en estados nación, es decir, por la preponderancia de la nacionalización y la estatalización de los fenómenos sociales (Santamaría, 1994). El estado nación determina quiénes son sus miem- 
bros (los nacionales) y quiénes los foráneos (los no nacionales, los extranjeros). Con todo, puede afirmarse que existen distintos grados de «foraneidad». Ello depende del país de origen de los extranjeros, de las causas que les han llevado a instalarse en España y, también, del grado de asentamiento de los grupos inmigrados (Delgado, 1992), lo que permite diferenciar al extranjero del inmigrante, del nouvingut (new comer) o recién llegado.

En cualquier caso, tal y como ocurre en Winston Parva, la implantación de la exterioridad viene determinada por las relaciones de poder entre la sociedad de acogida y el grupo de foráneos. Ello da lugar a un tipo social: el extranjero.

[...] el extranjero es una presencia provisional y duradera, a la vez que se define por no pertenecer a ese espacio social desde siempre y por incorporar cualidades que presuntamente no proceden ni pueden proceder de él. (Simmel, 1908)

Con lo cual, la atribución de dichas cualidades opera como un estigma y un principio denegatorio.

La definición del inmigrante como figura social está estrechamente relacionada con la estigmatización. Para ser inmigrante, no basta con ser el extranjero que viene de un país foráneo, hace falta ser un intruso no invitado y pobre, lo que nos remite al imaginario del inmigrante «irregular» procedente de países en vías de desarrollo. El inmigrante es estigmatizado también por su inferioridad en el plano cultural y su atraso en términos de civilización (Delgado, 1992). Es definido como una figura social «peligrosa» para la integridad del grupo y para su seguridad, con lo cual aparece aquí un claro paralelismo con la comunidad de Winston Parva, donde el grupo de antiguos residentes, cuyos miembros se conocían entre sí desde hacía generaciones, había forjado un modo de vida y un conjunto de normas compartidas, respetaba ciertos principios y se sentía orgulloso de ello. Esto era precisamente lo que le concedía el prestigio que a sí mismo se atribuía: tradición y vida comunal. La llegada de nuevos habitantes al vecindario se experimentaba como una amenaza a su modo de vida, aun siendo como eran los recién llegados compatriotas.

Igualmente, pueden establecerse distintos grados de «inmigridad» en relación con el lugar de origen y el tiempo de asentamiento. Mientras que los extranjeros procedentes de países pobres, que emigran como estrategia de supervivencia y lo hacen en situación administrativa irregular, serían inmigrantes absolutos, aquellos que provinieron de otras partes de España, como por ejemplo los andaluces o murcianos que emigraron a las diferentes provincias catalanas, y que están adaptados política y socialmente a Cataluña, aunque no culturalmente, serían inmigrantes relativos: forasteros, charnegos (Delgado, 1992).

A nivel nacional, la preservación de esta lógica tiene su base en la política de extranjería que define quién es extranjero y quién es, además, extranjero irregular, lo cual establece las lógicas de poder entre nacionales y no naciona- 
les, pero también entre inmigrantes regulares e irregulares, lo cual reconoce a un tiempo determinados derechos a unos y se los deniega a otros. Es importante considerar cómo todo ello ejerce una fuerte influencia, por un lado, en el grado de aceptación de las sociedades receptoras hacia el establecimiento de grupos de nacionalidades extranjeras en nuestras localidades y barrios, pero, por otro, en el desarrollo de las políticas de integración (Alexander, 2003).

\section{Conclusiones. Sugerencias para la investigación en el campo de la sociología de las migraciones desde la lectura de Elias}

La obra de Elias y Scotson incluye numerosos aspectos que, como se ha probado, habían venido siendo analizados por otros sociólogos anteriores y coetáneos a estos dos autores. Con todo, desde mi punto de vista, la obra de Elias aporta sugerentes elementos que complementan los estudios referenciados y que resultan ciertamente útiles para el estudio y el análisis sobre cómo se configuran las relaciones de convivencia en el espacio suburbano entre diferentes grupos.

Una característica que, a mi modo de ver, resulta relevante del estudio de Elias y Scotson es el hecho de que su análisis se focalice en una pequeña comunidad suburbana, lo que aporta una perspectiva centrada en la comunidad y en las relaciones personales y familiares que en ella se establecen.

En este sentido, frecuentemente, los estudios sobre inmigración suelen adoptar el análisis de los discursos y las prácticas de los autóctonos hacia los inmigrantes considerando a la sociedad de acogida en su conjunto y tomando la ciudad como un lugar genérico e impersonal en el que se producen las relaciones de convivencia ${ }^{2}$. Es por ello que resulta sugerente, a la luz de la obra que aquí hemos analizado, plantear el análisis de estas lógicas de estigmatización social y de alteridades teniendo en cuenta espacios suburbanos más acotados, como podría ser el barrio, y estudiarlo desde un análisis comunal.

Estimo que la investigación llevada a cabo en Winston Parva sugiere una línea de profundización escasamente desarrollada en los estudios sobre inmigración y convivencia, así como sobre las relaciones interétnicas. Por lo general, este tipo de investigaciones se focalizan en la consideración de dos grandes grupos: los españoles y los inmigrantes y las lógicas de discriminación o rechazo de los primeros hacia los segundos. Rara vez se aborda la cuestión de la convivencia entre e intra los diferentes grupos de inmigrados. La exploración de discursos y actitudes suele estar focalizada en los discursos y las actitudes de los españoles hacia los inmigrantes, sin considerar que los inmigrantes no tienen porqué ser grupos cohesionados por su condición nacional. Plantear el análisis desde la dimensión local y comunitaria aportaría, ciertamente, elementos reveladores sobre esta cuestión, dado que, en determinadas

2. Algunos ejemplos los encontramos en: IMSERSO, 1998; Zapata y Van Dijk, 2007; Echeverri Buriticá, Ibáñez y Ortí, 2007. 
localidades, la convivencia parece darse, precisamente, entre grupos de inmigrantes como resultado de los procesos de asentamiento de esta población en ciertas zonas de nuestras ciudades.

Igualmente, todas las encuestas de opinión sobre inmigración están orientadas a recoger qué opinan los españoles y las españolas de los inmigrantes. A nadie se le ha ocurrido preguntar qué opinan los inmigrantes sobre la inmigración. ¿¿Debemos suponer que los inmigrantes no son racistas? ¿No tienen sus xenofobias y sus xenofilias? ¿No son clasistas, acaso? ¿No defienden sus intereses frente a otros nuevos inmigrantes?

En este sentido, resulta interesante recordar cómo Elias y Scotson ponían de manifiesto que pertenecer al grupo de los established comportaba un precio: el de someterse a las normas del grupo, lo que evoca el control que pueden ejercer ciertas comunidades de inmigrantes sobre sus miembros. Un ejemplo claro sería el de las comunidades islámicas y el control sobre sus mujeres (Alcalde, 2002; Colectivo Ioé, 1998; Ramírez, 1998; Roque, 1999; Solé, 1994), por lo que parece pertinente estudiar qué normas son esas y cómo se estigmatiza a aquel o a aquella que no las cumple.

En el caso de los marroquíes, sería interesante estudiar las relaciones comunitarias en relación a su origen árabe o bereber, a sus tradiciones, a sus lenguas, etc. Pablo Pumares (1994) reconoce cómo se perciben con fuerza estas diferencias, que pueden verse acentuadas en la emigración en el extranjero con relación a costumbres concretas y lógicas de solidaridad (cohesiones de los grupos en base a formas de comportarse y costumbres). Igualmente, las influencias culturales no se difunden igual entre las diferentes clases sociales ni según el origen rural o urbano,

Igualmente, podrían aplicarse estas consideraciones a los grupos de inmigrantes chinos, tan ampliamente diversos en su composición también en un sentido étnico, religioso y lingüístico (Beltrán, 2005; Nieto, 2007).

En definitiva, la obra de Elias y Scotson plantea una cuestión que sería necesario indagar con mayor profundidad sobre la integración sociocultural de los inmigrados extranjeros residentes en España. A saber, las lógicas de discriminación intergrupales según el orden de llegada y las intragrupales, vinculadas a la observación de las normas y los valores que configuran al grupo, les otorga homogeneidad e identidad.

\section{Bibliografia}

AlCALDE, R. et al. (2002). «Las mujeres marroquíes en Cataluña: entre la transgresión y el cambio». Revista Catalana de Sociologia, 18, 27-44.

AleXAnder, M. (2003). «Local Policies Howard migrants as an expresión of HostStranger relations: a proponed typology». Journal of Ethnic and Migration Studies, $29(3), 411-430$.

BAumAN, Z. (1995). «Making and unmaking of Strangers». Thesis Eleven, 43 (1), 1-16. BÉJAR Merino, Helena (1994). «Norbert Elias, retrato de un marginado». Reis: Revista Española de Investigaciones Sociológicas, 65, 13-26. 
Beltrán, J. (2005). «Las comunidades asiáticas en España: una visión panorámica». Revista CIDOB d'Afers Internacionals, 33-51.

Echeverri Buriticá, M.M.; IbáÑez Rojo, R. y Ortí Mata, M. (2007). El codesarrollo desde la perspectiva de la población inmigrante. Informe de investigación: una aproximación a las representaciones sociales y discursos sobre el desarrollo de las y los inmigrantes marroquíes y ecuatorianos en Madrid. Madrid: Asociación para la Cooperación con el Sur-Las Segovias.

Colectivo Ió́ (1995). «Discurso de los españoles sobre los extranjeros: Paradojas de la alteridad. Informe (resultados del estudio 2119)». Opiniones y Actitudes, 8.

- (1998). "Mujeres inmigrantes en España. Proyectos migratorios y trayectorias de género". Revista Ofrim. Suplementos, 3, 11-37.

Elias, N. y SCOTSON, J.L. (1994). The stablised and the outsider. Londres: Sage Publication (1965).

ELIAS, N. (2003). «Ensayo acerca de las relaciones entre establecidos y forasteros». Reis, 104, 219-251.

Delgado Ruiz, M. (1992). Ciutat i immigració. Barcelona: CCCB.

GOFFMAN, I. (1993). La presentación de la persona en la vida cotidiana. Buenos Aires: Amorrortu (1950).

- (2003). Estigma: La identidad deteriorada. Buenos Aires: Amorrortu (1963).

IMSERSO (1998). Actitudes hacia los inmigrantes. Madrid: IMSERSO. Colección Observatorio Permanente de la Inmigración, 2.

MartíneZ, E. (2000). «Estudio: Migraciones, cambios sociales e híbridos culturales». Scripta Nova: Revista Electrónica de Geografía y Ciencias Sociales, 75. [http://www.ub.es/geocrit/sn-75.htm]

NieTO, G. (2007). La inmigración china en España: una comunidad ligada a su nación. Madrid: Los Libros de la Catarata.

PARK, R.E. (1914). "Racial Assimilation in Secondary Groups». Publications of the American Sociological Society, 8.

- (1928). "Human Migrations and the Marginal Man». Publicado originalmente en American Journal of Sociology, 1928 (mayo), 33, 881-893. Recogido posteriormente en el libro recopilatorio de Robert E. PARK, On Social Control and Collective Behavior, editado por Ralph H. Turner. Chicago: The University of Chicago Press, 1967, 194-206.

PEÑA, G. de la (2003). «Simmel y la Escuela de Chicago en torno a los espacios públicos en la ciudad». Sincronía, 3. [http://sincronia.cucsh.udg.mx/pena03.htm]

Pumares, P. (1994). La inmigración marroqui en la Comunidad de Madrid. Tesis doctoral dirigida por José Estébanez Álvarez. Universidad Complutense de Madrid.

Ramírez, A. (1998). Migraciones, género e islam: Mujeres marroquíes en España. Madrid: Agencia Española de Cooperación Internacional.

Roque, Ma A. (dir.) (1999). Dona i migració a la Mediterrània occidental. Barcelona: Institut Català de la Mediterrània d'Estudis i Cooperació.

SANTAmaría, E. (1994). "Extranjero", nada menos que una palabra mayor». Papers: Revista de Sociología, 43, 63-70.

- (2002). «Inmigración y barbarie: la construcción social y política del inmigrante como amenaza». Papers: Revista de Sociologia, 66, 59-75.

- (2002). La incógnita del extraño: Una aproximación a la significación sociológica de la «inmigración» no comunitaria. Barcelona: Anthropos.

SCHÜTZ, A. (1974). «El forastero». En: Estudios sobre teoría social. Buenos Aires: Amorrortu (1944, 1946). 
Simmel, G. (1908). «The stranger». En: WolfF, K.H. (transl. y ed.) (1950). The Sociology of Georg Simmel. Nueva York: Free Press, 402-6.

- (1977). "Digresiones sobre el extranjero». Sociología, II. Revista de Occidente. Madrid: (1927).

SolÉ, C. (1994). La mujer inmigrante. Madrid: Instituto de la Mujer.

Thomas, W.I. y ZnANIECKI, F. (1918). The polish peasant in Europe and America. Boston: Badger.

Zapata Barrero, R.; VAN DijK, T. (ed.) (2007). Discursos sobre la inmigración en España: Los medios de comunicación, los parlamentos y las administraciones. Bellaterra: Ediciones Bellaterra. 